Espacio y Desarrollo No33, 2019, pp. 91-116 (ISSN 1016-9148)

https://doi.org/10.18800/espacioydesarrollo.201901.005

\title{
Avances y retos en el accionar del Ministerio de Ambiente y Desarrollo Sostenible para controlar la Deforestación EN la Amazonía COLOMbiana
}

\author{
Ivonne Otero-Durán* \\ Universidad de los Andes, Colombia \\ i.otero@uniandes.edu.co \\ Maricel Piniero** \\ Agrosavia - Corporación Colombiana de Investigación Agropecuaria \\ mpiniero@gmail.com
}

Fecha de recepción: 1 de abril de 2109

Fecha de aceptación: 24 de mayo de 2019

\section{Resumen}

El Ministerio de Ambiente y Desarrollo Sostenible (MADS) es la entidad rectora de la gestión de los recursos naturales de Colombia, que define políticas y regulaciones para asegurar el desarrollo sostenible. Sin embargo, los conflictos ambientales son persistentes y la deforestación está aumentando, poniendo en riesgo el logro de la meta de reducir a cero la deforestación neta en la Amazonía para 2020, definida en el marco del Acuerdo de París sobre cambio climático y en la estrategia de implementación de los Objetivos de Desarrollo Sostenible (ODS). En este contexto, se analizó al MADS para comprender de qué forma opera para controlar la deforestación y contribuir con el desarrollo sostenible en la Amazonía colombiana, y se indagó acerca de las dificultades que esta entidad afronta para lograrlo. Si bien existen instrumentos normativos y propuestas de intervención, la contradicción entre las políticas sectoriales y los problemas de orden público son, entre otros, factores que dificultan la implementación de modelos de desarrollo sostenible y el control de la deforestación en el país.

Palabras clave: conservación, deforestación, desarrollo sostenible, institución.

* Bióloga, magíster en Estudios Interdisciplinarios sobre Desarrollo y especialista en Planeación Ambiental. Consultora en temas de gestión ambiental, gobernanza y desarrollo.

** Investigadora en Agrosavia - Corporación Colombiana de Investigación Agropecuaria. 


\title{
Advances and challenges in the actions of the Ministry of Environment and Sustainable Development to control deforestation in the Colombian Amazon
}

\begin{abstract}
The Ministry of Environment and Sustainable Development (MADS) is the governing entity for the management of natural resources in Colombia, which defines policies and regulations to guarantee sustainable development. Nevertheless, environmental conflicts are persistent, and deforestation is increasing, putting the achievement of the goal of zero net deforestation in the Amazon by 2020 (established within the framework of the Paris Agreement on climate change and the strategy of implementation of the Sustainable Development Goals [SDGs]) at risk. In this context, the MADS was analysed to understand how it operates to control deforestation and contribute to sustainable development in the Colombian Amazon, and it was inquired about the difficulties that this entity faces to achieve it. Although there are normative instruments and proposals for intervention, the contradiction between sectoral policies and problems of public order are, among others, factors that hinder the implementation of models of sustainable development and the control of deforestation in the country.
\end{abstract}

Keywords: conservation, deforestation, sustainable development, institution.

\section{INTRODUCCIÓN}

La visión de desarrollo en Colombia está orientada por los principios del desarrollo sostenible, como se evidencia en la Constitución Política de Colombia (1991). Esta visión es visible en las políticas, planes y proyectos de desarrollo del Estado y constituye un insumo clave para avanzar en las metas trazadas dentro de la Estrategia para la implementación de los Objetivos de Desarrollo Sostenible (ODS) en Colombia (Departamento Nacional de Planeación [DNP], 2018a). De manera similar, la Sentencia STC4360-2018, que reconoce a la Amazonía colombiana como entidad «sujeto de derechos», ratifica el compromiso de la nación por contribuir con los ODS al reducir la deforestación en la Amazonía y garantizar los derechos ambientales de las generaciones futuras (Corte Suprema de Justicia, 2018).

Una de las apuestas del gobierno nacional para conducir al país hacia el desarrollo sostenible incluyó la creación del Ministerio de Ambiente y Desarrollo Sostenible (MADS), como el organismo encargado de impulsar una relación de respeto y armonía entre humanos y naturaleza y de definir las políticas y regulaciones necesarias para asegurar el desarrollo sostenible de la nación (Ley 99 de 1993, artículo 2). No obstante, pese a la existencia del MADS y a una Política Nacional para la Gestión Integral de la Biodiversidad y sus Servicios Ecosistémicos [PNGIBSE] (MADS, 2012), la pérdida de biodiversidad y servicios ecosistémicos es un fenómeno permanente en el país debido a actividades que generan cambios en el uso del suelo y degradación de los ecosistemas; 
tales como la infraestructura, la expansión de la frontera agropecuaria, la expansión del sector minero, la urbanización y el cambio climático (MADS y PNUD, 2014). Por lo que respecta específicamente a la deforestación, las cifras del MADS (2015) indican que entre 1990 y 2010 se perdieron cerca de 2,2 millones de hectáreas de Selva Amazónica, a tasas de entre 80 mil y 120 mil ha por año; y de acuerdo con el Instituto Amazónico de Investigaciones Científicas (Sinchi) (2016) en el período 2010 - 2013 fueron deforestadas 140000 hectáreas de bosque al año. En 2016 fueron deforestadas 178597 ha de bosque (Instituto de Hidrología, Meteorología y Estudios Ambientales [Ideam] 2016, citado por Sistema de Información Ambiental de Colombia [SIAC], 2016) y en 2017 la cifra aumentó a 219973 ha (Ideam, 2018).

Ante esta situación, actualmente el MADS lidera la implementación de «Visión Amazonía», una alianza entre los gobiernos de Colombia, Alemania, Noruega y el Reino Unido, que busca reducir a cero la deforestación neta en la Amazonía colombiana para 2020 a través de la promoción de un nuevo modelo de desarrollo sostenible que, al mismo tiempo, mejore la calidad de vida de las personas y mantenga la biodiversidad (MADS, 2016). La implementación de acciones para el control de la deforestación en la Amazonía se fundamenta en que el 65,5\% de la deforestación nacional en 2017 ocurrió en esa región, principalmente en los departamentos de Caquetá (con 56113 ha deforestadas en los municipios de San Vicente del Caguán, Cartagena del Chairá, y Solano) y Guaviare (con 37044 ha deforestadas en los municipios de San José del Guaviare, Calamar y El Retorno) (IDEAM, 2017). Lo cual se debe, en gran medida, a un proceso de acaparamiento de tierras que implica la praderización, principalmente para el desarrollo de la actividad ganadera (MADS, 2018a).

En este orden de ideas y frente a la alarmante situación mencionada anteriormente, surge la necesidad de comprender de qué forma el MADS trabaja para controlar la deforestación y contribuir con el desarrollo sostenible en Colombia, particularmente en aquellos territorios con altas tasas de deforestación. Con este propósito, en esta investigación se plantearon tres objetivos: identificar cómo es entendido el concepto de desarrollo sostenible dentro del MADS y cómo se ha incorporado en el accionar del MADS, analizar las intervenciones del MADS para promover el desarrollo sostenible como mecanismo para reducir la deforestación en la Amazonía colombiana, e identificar fortalezas y debilidades en la estrategia que el MADS implementa para contribuir con el desarrollo sostenible en la Amazonía colombiana.

Así, para realizar la investigación se analizó al MADS viéndolo como institución y como organización, tomando como referente una de las conclusiones del análisis de Barrera, Bedoya y Rodríguez (2007), quienes plantearon que los ministerios deben contemplarse desde una doble perspectiva: como institución, incluyendo las normas, prácticas y relaciones; y como organización, teniendo en cuenta el conjunto de recursos humanos, físicos y financieros que los integran. 
En consecuencia, resultó importante analizar la forma organizativa del MADS y las relaciones que establece con otras organizaciones, para comprender cómo esta entidad ejerce su rol, tanto en la generación de instrumentos de política como en la puesta en marcha de las estrategias encaminadas al desarrollo sostenible en los diferentes niveles territoriales. Este análisis se realizó a la luz del enfoque de desarrollo sostenible; haciendo especial énfasis en el decimoquinto objetivo de desarrollo sostenible (ODS 15): «Promover el uso sostenible de los ecosistemas terrestres, luchar contra la desertificación, detener e invertir la degradación de las tierras y frenar la pérdida de la diversidad biológica» (Unesco, 2015a). Al respecto, Gulick (1993) afirma que en el ámbito gubernamental es fácil encontrar ejemplos de resultados de combinaciones administrativas que dificultan conseguir eficacia técnica y, por ende, imposibilitar el logro de los objetivos de la organización. Y, si bien no existe una forma organizativa óptima que satisfaga las necesidades de todas las organizaciones (Avendańo, Fonseca y Marín, 2011), el estudio de la estructura organizativa del MADS es necesario para comprender el modo en el que este opera para promover modelos de desarrollo sostenible que reduzcan la deforestación en la Amazonía colombiana.

La presente investigación incluyó el análisis de la documentación relacionada con informes técnicos, normas, políticas y procesos del MADS, así como de la percepción de algunos funcionarios y contratistas sobre el accionar de la entidad en lo relacionado con la deforestación y el desarrollo sostenible. La información documental analizada fue recolectada entre los meses de enero de 2017 y marzo de 2018, por lo cual los datos presentados en el documento no reflejan de manera significativa los cambios coyunturales relacionados con la generación de instrumentos normativos posteriores a esta fecha, tales como la Política de Crecimiento Verde (DNP, 2018b), el reconocimiento de la Amazonía colombiana como entidad «sujeto de derechos» (Corte Suprema de Justicia, 2018), ni la definición de la frontera agrícola nacional (Ministerio de Agricultura y Desarrollo Rural [MADR], 2018). De manera similar, dado que las entrevistas fueron implementadas entre el 12 de marzo y el 5 de abril de 2018, tampoco se reflejan cambios de percepción de los entrevistados tras el surgimiento de las normas anteriormente mencionadas.

\section{EL DESARROLLO SOSTENIBLE COMO MARCO DE ANÁLISIS DE LAS INTERVENCIONES}

La búsqueda de estrategias para armonizar la conservación de los ecosistemas y el desarrollo se remonta a la década de 1970, cuando se realizó la primera Conferencia Internacional sobre el Medio Humano, también denominada "Conferencia de Estocolmo", que generó un llamado internacional para trabajar en torno a la conservación y evitar la degradación ambiental (Naciones Unidas, 1973). Más adelante, durante la Comisión Mundial sobre el Medio Ambiente y el Desarrollo (Comisión de Brundtland), 
se planteó la necesidad de cambios de actitudes, de valores sociales y de aspiraciones, para satisfacer las necesidades del presente salvaguardando los intereses de las generaciones futuras (United Nations, 1987); acuñando así el concepto «desarrollo sostenible», como aquel «desarrollo que satisface las necesidades de la generación presente, sin comprometer la capacidad de las generaciones futuras de satisfacer sus propias necesidades» (Unesco, 2015b, p. 1). El concepto de desarrollo sostenible planteado en la Comisión de Brundtland fue acogido e incorporado en la normativa colombiana desde 1991 cuando la Constitución Política estableció que «el Estado planificará el manejo y aprovechamiento de los recursos naturales, para garantizar su desarrollo sostenible» (Constitución Política de Colombia, 1991, artículo 80); y ratificado en la Ley 99 de 1993, en cuyo artículo 3 lo definió como:

el que conduzca al crecimiento económico, a la elevación de la calidad de la vida y al bienestar social, sin agotar la base de recursos naturales renovables en que se sustenta, ni deteriorar el medio ambiente o el derecho de las generaciones futuras a utilizarlo para la satisfacción de sus propias necesidades.

Recientemente, en 2015, la Organización de la Naciones Unidas adoptó los Objetivos de Desarrollo Sostenible (ODS) y estableció que las acciones enmarcadas en los ODS deberán buscar el progreso económico, social y tecnológico en armonía con la naturaleza (United Nations, 2015a). Colombia, como miembro de la ONU, fijó indicadores y metas relacionados con cada ODS y definió la estrategia de implementación de los ODS en el territorio nacional (DNP, 2018a).

Sin embargo, pese a que el término «desarrollo sostenible» y los ODS han traspasado fronteras y se ha buscado su incorporación en las políticas y programas de las diferentes naciones, su implementación ha presentado obstáculos; lo cual es evidente en las cifras de pérdida de biodiversidad alrededor del mundo por actividades relacionadas con el desarrollo económico, como lo son la infraestructura y la expansión de la frontera agropecuaria. Esta situación sugiere la necesidad de un análisis de la interpretación y puesta en marcha del término; el cual, tal como lo han planteado diversos autores (Naredo, 1987; Ehrlich, 1989; Escobar, 2011), puede resultar ambiguo, pesto que el concepto de crecimiento económico (o desarrollo) concebido por los economistas está separado del medio físico. Esto, dado que el desarrollo económico se mide de acuerdo con el aumento de los agregados monetarios haciendo abstracción de los procesos que los generan (Naredo, 1987). De acuerdo con esto, el término "desarrollo sostenible» puede ser visto como una utopía (Parada y Sánchez, 2014), hasta tanto no se generen cambios de prácticas y de visiones que permitan lograr transformaciones de fondo en el modelo actual de desarrollo (capitalista y antropocentrista), como mecanismo para salir de las condiciones críticas (Escobar, 2015). 


\section{El institucionalismo para el análisis del MADS}

El Sistema Nacional Ambiental (SINA) es el conjunto de orientaciones, normas, actividades, recursos, programas e instituciones que permiten la puesta en marcha de la política ambiental colombiana (Ley 99 de 1993, artículo 4); y es el MADS la institución de mayor jerarquía dentro del SINA. Teniendo en cuenta que el MADS es responsable de definir políticas y regulaciones para asegurar el desarrollo sostenible en el país, resulta necesario conocer a la entidad como institución y como organización, de acuerdo con la recomendación de Barrera et al. (2007), presentada en la introducción de este artículo.

Existen diversas connotaciones sobre las instituciones que las definen desde formas sociales de carácter universal — como la familia y la iglesia - hasta formas sociales dotadas de una organización jurídica y material, como las empresas (Barrera et al., 2007). Según North (1991), las instituciones son las reglas del juego formales e informales que regulan la interacción humana, proporcionando una estructura de incentivos que inciden en la conducta de los individuos. Las instituciones formales son reglas claramente definidas, como las leyes; mientras que las instituciones informales son acuerdos y códigos de conducta ideados por las personas, pero no establecidos por ley. Así puede suceder que el Estado intente imponer reglas externas frente al manejo de los recursos, pero que las personas sean quienes logren crear sus propios arreglos institucionales obligatorios para manejarlos efectivamente (Ostrom, 1990). Chevallier (1996) por su parte, entiende las instituciones como el conjunto de hechos sociales que tienen la apariencia de una realidad objetiva y que son asumidos como tales por los individuos; existiendo variedad de formas institucionales políticas y administrativas, que actúan en respuesta a problemas particulares.

Las organizaciones son «entidades que tienen el propósito de maximizar su utilidad, definida por las oportunidades que brinda la sociedad en el marco institucional» (North, 1993, p. 99). Estas están conformadas por un «conjunto de individuos que persiguen un fin común» (Alpuche de la Cruz y Bernal, 2015, p. 4), el cual varía en función del objetivo y del carácter (público o privado) de la organización. Así, de acuerdo con Alpuche de la Cruz y Bernal (2015), las organizaciones públicas cumplen con un mandato social y con referentes institucionales; han sido creadas para atender necesidades sociales, regulatorias, normativas y de apoyo; sus recursos provienen principalmente del presupuesto público; y su viabilidad está basada en el grado de cumplimiento con la sociedad.

De acuerdo con lo anterior, para efectos de esta investigación, se parte del hecho de que el MADS, como institución administrativa que hace parte del arreglo institucional denominado «Estado», y las personas que lo integran, han asumido funciones en el conjunto de la institucionalidad pública en aras de cumplir con el propósito de llevar a 
Colombia hacia un desarrollo sostenible. Por consiguiente, el análisis de la concepción e incorporación del concepto de desarrollo sostenible en el MADS, sumado al análisis de la entidad como institución y como organización, orienta la comprensión de su accionar en el territorio para reducir la deforestación y para la promoción de modelos de desarrollo sostenible en la Amazonía.

\section{Metodología}

La estrategia metodológica adoptada en este trabajo corresponde a un estudio de caso de tipo instrumental (Stake, 1995, citado por Creswell, 2013). La investigación, de carácter cualitativo, se desarrolló en fases: la primera consistió en la revisión y análisis de información disponible acerca del objeto de estudio; esta fue sucedida por una segunda fase que incluyó la aplicación de entrevistas semiestructuradas a actores claves para la investigación. Por último, se realizó el análisis de los datos de manera descriptiva, buscando patrones, similitudes y diferencias para responder la pregunta de investigación. En el análisis de los documentos técnicos se indagó acerca de la inclusión del término "desarrollo sostenible / sustentable» de manera explícita en los textos, pero también se buscó si se hace referencia a otros elementos que aportan al desarrollo sostenible tales como: 1) uso, manejo o aprovechamiento sostenible, 2) conservación de la biodiversidad y los servicios ecosistémicos, 3) gobernanza, 4) calidad de vida o bienestar y 5) cultura o medios de vida.

La selección de los entrevistados se realizó inicialmente a través de un muestreo intencional, el cual fue complementado con un muestreo en avalancha dado que los primeros entrevistados recomendaron a otras personas que ellos consideran claves para la investigación (Martín-Crespo y Salamanca, 2007). Así pues, fueron aplicadas 11 entrevistas al interior del MADS, con una duración promedio de 45 minutos, a profesionales y contratistas (incluyendo niveles directivos y técnicos) que participan o han participado en proyectos relacionados con el control de la deforestación o la generación de propuestas encaminadas al desarrollo sostenible en el país. La muestra incluyó profesionales de tres áreas del MADS: la Dirección de Bosques, Biodiversidad y Servicios Ecosistémicos (DBBSE), la Oficina Asesora de Planeación y la Oficina de Negocios Verdes y Sostenibles. Se emplearon seudónimos para proteger la identidad de aquellos entrevistados que solicitaron anonimato.

Las preguntas realizadas a los entrevistados incluyeron: ¿Qué es para usted la «conservación» ?; ¿Qué es para usted el «desarrollo sostenible» ?; ¿Existe una definición del concepto «desarrollo sostenible» adoptada por el MADS? En caso afirmativo, ¿̨uál es esa definición?; Mencione los números de los ODS que hacen parte de la estrategia para el control de la deforestación en la Amazonía colombiana; ¿Cómo incorpora el desarrollo sostenible en la formulación de proyectos?; ¿¿De qué manera los proyectos 
le apuntan al desarrollo sostenible?; ¿Qué porcentaje de los proyectos que se formulan en el MADS incluyen mecanismos de monitoreo?; ¿Con cuáles organizaciones (públicas y privadas) trabaja el MADS para la reducción de la deforestación en la Amazonía?; ¿Existe articulación entre las diferentes dependencias del MADS para formular los proyectos?. ¿Cuáles son las principales dificultades para que las acciones implementadas conduzcan al logro del desarrollo sostenible y a la reducción de la deforestación en la Amazonía colombiana? Las respuestas fueron codificadas inicialmente empleando frases descriptivas simples y, posteriormente, organizadas en categorías de análisis (Creswell, 2014) para, finalmente plantear conclusiones.

\section{Resultados}

\subsection{La estructura organizacional del MADS y su accionar institucional}

El MADS es, por ley, la entidad responsable de definir las políticas y regulaciones a las que se sujetarán la recuperación, la conservación y el manejo de los recursos naturales renovables y el medio ambiente de la Nación, para asegurar el desarrollo sostenible (Ley 99 de 1993, Art. 2). En su visión institucional el MADS establece que la entidad en 2020 «fomentará el desarrollo sostenible del país (...) y será líder de la educación ambiental y articulador entre el sector productivo y los diferentes actores para la implementación y seguimiento a las políticas y lineamientos ambientales» (MADS, s.f).

En los últimos años, y acorde con lo establecido en el artículo 16 del Decreto 3570 de 2011 sobre las funciones del MADS, este ha formulado directrices, instrumentos de política y programas orientados a contribuir con la conservación y uso sostenible de los bosques (Tabla 1) y ha participado en la formulación de documentos de política de manera articulada con el Departamento Nacional de Planeación (DNP) y otros ministerios.

Así, en la PNGIBSE se estableció que la biodiversidad debe «ser el principal elemento estructurador de los procesos de ordenamiento territorial, al ser fuente y garantía del suministro de servicios ecosistémicos claves para el desarrollo y la sostenibilidad de las actividades humanas de producción, extracción, asentamiento y consumo» (MADS, 2012, p. 40). Además, con la formulación del Plan Nacional de Restauración, el MADS orienta y promueve la restauración ecológica, la recuperación y la rehabilitación de áreas disturbadas de Colombia lo cual contribuye de manera directa con el logro de la meta para 2020 del indicador de pérdida anual de bosques naturales definida para el ODS 15: deforestación neta cero. Esto, teniendo en cuenta que la meta hace referencia al balance entre la conversión de bosques a otros usos de la tierra y el paso de otros usos de la tierra a bosques (a través de procesos de restauración), que sumados equivalen a cero. 
Tabla 1. Principales instrumentos generados por el MADS entre 2012 y 2018, que contribuyen con el control de la deforestación

\begin{tabular}{lll}
\hline Documento / Estrategia & Fecha & Descripción general \\
\hline $\begin{array}{l}\text { Política Nacional para la Gestión } \\
\text { Integral de Biodiversidad y sus } \\
\text { Servicios Ecosistémicos - PNGIBSE }\end{array}$ & 2012 & $\begin{array}{l}\text { Promueve la Gestión Integral de la Biodiversidad } \\
\text { y sus Servicios Ecosistémicos de manera que se } \\
\text { mantenga y mejore la resiliencia de los sistemas } \\
\text { socio-ecológicos. }\end{array}$ \\
\hline
\end{tabular}

Define lineamientos para impulsar actividades

Plan Nacional de Negocios Verdes $2014 \quad$ económicas que incorporan buenas prácticas ambientales, sociales y económicas (negocios verdes).

Orienta y promueve la restauración ecológica, la recuperación y la rehabilitación de áreas Plan Nacional de Restauración 2015 disturbadas de Colombia en un marco amplio de conservación de la biodiversidad y la adaptación a los cambios globales.

Busca que la madera extraída, transportada, Pacto intersectorial por la madera legal 2015 transformada, comercializada y utilizada provenga exclusivamente de fuentes legales.

Programa de trabajo con un enfoque de desarro-

Programa Visión Amazonía

2015 llo regional con baja deforestación que fortalezca la gobernanza.

Esquema de Reconocimiento a la

Procedencia Legal y de Promoción

hacia el Manejo Sostenible en el 2016

Busca incentivar a los usuarios del bosque natural

Aprovechamiento Forestal de Bosques a que desarrollen actividades de aprovechamiento

Naturales

sostenible de los recursos forestales.

Plan de Acción de Biodiversidad

2016-2030

\begin{tabular}{ll}
\hline Resolución 0470 & $\begin{array}{l}\text { Febrero } \\
\text { de } 2017\end{array}$ \\
\hline Decreto 1257 & $\begin{array}{l}\text { Julio de } \\
2017\end{array}$
\end{tabular}

Decreto 1655

Octubre

de 2017
Promueve la incorporación de la biodiversidad y sus servicios ecosistémicos en la planificación sectorial y promueve su aprovechamiento sostenible.

Crea el Programa «Bosques de Paz» como modelo de gestión sostenible de los territorios.

Crea la Comisión Intersectorial para el Control de la Deforestación y la Gestión Integral para la Protección de Bosques Naturales - CICOD.

Organización y funcionamiento del Sistema Nacional de Información Forestal, el Inventario Forestal Nacional y el Sistema de Monitoreo de Bosques y Carbono que hacen parte del Sistema de Información Ambiental para Colombia.

Mayo de Estrategia Integral de Control a la Deforestación 2018 y Gestión de los Bosques.

Junio de Reglamenta el incentivo de pago por servicios 2018 ambientales en áreas y ecosistemas estratégicos. 
Adicionalmente, el MADS actualmente implementa el pacto intersectorial por la madera legal, el cual ha sido firmado por 73 organizaciones (tanto públicas como privadas). Esta iniciativa está articulada con la estrategia de crecimiento verde contenida en el Plan Nacional de Desarrollo 2014-2018, cuyos objetivos incluyen la protección y el aseguramiento del uso sostenible del capital natural y el mejoramiento de la calidad y la gobernanza ambiental (DNP, 2014); poniendo en evidencia que el MADS está incorporando elementos que robustecen el concepto de desarrollo sostenible y contribuyen con su operatividad en el país.

De manera similar, la generación de once instrumentos orientados a apoyar la consolidación de la gobernanza forestal, tales como el Esquema de Reconocimiento a la Procedencia Legaly de Promoción hacia el Manejo Sostenible en el Aprovechamiento Forestal de Bosques Naturales, revelan el rol del MADS como una institución que implementa incentivos orientados a generar cambios de conducta, tal como lo sugiere North (1991) cuando define las instituciones.

El documento de política más reciente producido por el MADS es Bosques Territorios de Vida, que contiene la Estrategia Integral de Control a la Deforestación y gestión de los bosques (EICD), la cual define una ruta de trabajo para controlar la deforestación y las emisiones de gases efecto invernadero (GEI), contemplando elementos biofísicos y socioeconómicos (MADS, 2018a).

Ahora bien, para cumplir sus funciones, el MADS cuenta con una estructura organizacional en la que los proyectos son desarrollados a través de dependencias. El despacho del viceministro incluye cuatro direcciones, dentro de las que se encuentra la DBBSE que tiene la responsabilidad de aportar los elementos técnicos para la elaboración de la política y la regulación de la biodiversidad; aportar los elementos técnicos para la formulación del Plan Nacional de Desarrollo Forestal y coordinar su implementación; definir y orientar la implementación de la estrategia nacional de reducción de emisiones por deforestación y degradación de bosques; y proponer las bases técnicas para la regulación de las condiciones generales del uso sostenible, aprovechamiento, manejo, conservación y restauración de la diversidad biológica. Esta dirección cuenta con un grupo específico responsable de liderar las acciones relacionadas con la reducción de la deforestación, lo cual realiza a través de cinco ejes temáticos: gobernanza forestal, control a la deforestación, reservas forestales, ordenación forestal y restauración. El equipo técnico de la DBBSE participa en actividades que incluyen la formulación de planes, programas y proyectos, el acompañamiento y apoyo a los espacios de diálogo y de concertación en campo y el seguimiento a la implementación de las acciones.

Para ejercer sus funciones, el MADS se articula con diferentes organizaciones, nacionales y extranjeras, tanto del sector público como privado, quienes cumplen roles que van desde la cofinanciación de los programas (como es el caso de los proyectos con cooperación internacional y los convenios interadministrativos) hasta la implementación 
de acciones en campo. Es decir que, si bien el MADS es responsable de dar lineamientos y de orientar los procesos que las Corporaciones Autónomas Regionales (CAR) y las ONG implementan en los territorios, y no de desarrollar los procesos de manera directa en campo, el equipo técnico del MADS participa en espacios de generación de política pero también se involucra en las intervenciones y llega a trabajar directamente con la comunidad en algunos escenarios. Esta cercanía con la comunidad, de un modo más horizontal que vertical, es evidente en el acompanamiento específico que uno de los profesionales del equipo de Bosques realiza desde hace más de cuatro años a las diez mesas forestales departamentales que, en el marco del Proyecto de Consolidación de la Gobernanza Forestal, se han conformado en Colombia.

El MADS está trabajando no solo con organizaciones del sector ambiental, sino que está interactuando con entidades del sector económico en aras de generar mecanismos conducentes a reducir la reforestación. Al respecto, para evitar la conversión de los ecosistemas naturales en terrenos de uso agropecuario y reducir la deforestación tropical asociada con el aprovisionamiento de productos como el aceite de palma, la carne vacuna, el papel y pulpa ${ }^{1}$, Colombia constituyó recientemente la Alianza TFA 2020, orientada a «articular proyectos e iniciativas para promover la transformación positiva de las cadenas de suministro de materias primas agropecuarias hacia la cero deforestación, contribuyendo a la reducción de la deforestación en Colombia» (Tropical Forest Alliance 2020, 2017, p. 3); lo cual es un avance de articulación interinstitucional muy importante, si se tiene en cuenta que el sector Agricultura, Silvicultura y Otros Usos de la Tierra (Afolu) es el principal responsable de la deforestación en América latina (Tropical Forest Alliance 2020, 2017).

La relación con las autoridades ambientales, particularmente con las CAR, consiste en dar lineamientos acerca del modo para intervenir en los territorios, brindando asesoría técnica general para la implementación de acciones. Al mismo tiempo las CAR deben reportar unos indicadores de gestión al MADS. De manera similar, la relación con gobernaciones y con alcaldías se enfoca en hacerlos partícipes de los proyectos en el territorio, para fortalecer la implementación y seguimiento y para generar confianza entre los actores comunitarios que se vinculan a los procesos.

Los institutos de investigación y las universidades son responsables de generar información técnica de soporte para la toma de decisiones del MADS al mismo tiempo que formulan propuestas para la gestión territorial. Así, por ejemplo, el instituto SINCHI hace parte del pilar agroambiental del programa Visión Amazonía en donde implementa programas de mejoramiento de prácticas y de aprovechamiento de frutos secundarios del bosque.

\footnotetext{
1 De acuerdo con los compromisos adquiridos en la Declaración de Nueva York sobre Bosques de 2014 (United Nations, 2015b).
} 
Se destaca dentro de estas interacciones la relación que ha establecido con las comunidades (campesinas, indígenas y afros), así como con los gremios y empresas privadas, puesto que se maneja una interacción horizontal en la que se busca la articulación entre saberes y prácticas para el diseño de estrategias benéficas tanto para los ecosistemas como para las economías locales. De manera similar, el trabajo con las ONG permite mayor presencia en el territorio y facilita el acercamiento con los diferentes actores, así como la transmisión de la información en doble vía entre lo local, lo regional y lo nacional. Este es el caso del trabajo que desarrolla actualmente el Fondo Patrimonio Natural en el marco del pilar de gobernanza forestal del programa Visión Amazonía, quien realiza ejercicios de construcción colectiva de propuestas de desarrollo sostenible con las comunidades rurales de los departamentos de Caquetá, Guaviare y Meta. Del mismo modo, gracias al desarrollo de talleres de diagnóstico rural participativo realizado por la World Wildlife Fund (WWF), fue posible identificar los principales motores de deforestación en Putumayo (WWF, 2015). Las acciones participativas con diferentes tipos de actores sociales permiten la generación de proyectos desde la base, incorporando los intereses y visiones de las comunidades y motivándolos a participar, lo cual incrementa las posibilidades de permanencia en el tiempo de las acciones. $\mathrm{Al}$ respecto Dayer, Lutter, Sesser, Hickey y Gardali (2017) afirman que uno de los elementos que afecta la persistencia de las acciones implementadas tras la finalización de un programa es la falta de motivación; lo cual ocurre cuando se plantean acciones con las que las comunidades no se sienten a gusto o con las cuales no perciben beneficios.

Por lo que respecta a la articulación con actores internacionales se destaca el intercambio de conocimiento y la financiación para proyectos que en ausencia de fuentes económicas externas no podrían llevarse a cabo. Además, es importante tener en cuenta que la financiación extranjera se realiza a través del esquema de pago por resultados, lo cual constituye un mecanismo que contribuye a hacer más riguroso el trabajo del MADS.

\subsection{La incorporación del enfoque de desarrollo sostenible en las intervenciones del MADS}

Los documentos técnicos del MADS y las concepciones de los funcionarios y contratistas entrevistados ponen de manifiesto que el concepto de desarrollo sostenible adoptado en el país a través de la Ley 99 de 1993 ha evolucionado en la entidad, del mismo modo que el concepto ha ido evolucionando en el contexto internacional; y que, en la actualidad, es concebido como algo mucho más amplio teniendo en cuenta la realidad territorial. Esta nueva concepción incorpora elementos como el aprovechamiento sostenible, la articulación de múltiples visiones y sectores, el biocomercio sostenible, la gobernanza, la participación y la cultura. Lo anterior es visible, por ejemplo, en la formulación de los pilares de Visión Amazonía (gobernanza forestal, desarrollo y planeación sostenible sectorial, agroambiental, gobernanza ambiental con pueblos indígenas) 
y en la EICD, cuyos objetivos específicos uno y dos se orientan hacia la consolidación de la gobernanza forestal de grupos étnicos, comunidades campesinas y rurales; hacia el fortalecimiento de la conciencia ciudadana y la corresponsabilidad; y hacia el fomento de una economía forestal que contempla el aprovechamiento sostenible de los bosques.

Por lo que respecta a las entrevistas, cabe resaltar que las respuestas de todos los entrevistados, desde aquellos que ocupan los niveles directivos hasta los que se desempeñan en el nivel técnico, incorporan elementos complementarios a la definición de desarrollo sostenible adoptada en el país. Así, por ejemplo, el análisis de las respuestas de los entrevistados a las preguntas relacionadas con los conceptos "conservación» y "desarrollo sostenible» y con su incorporación en los instrumentos que ellos formulan, reflejan la complejidad del desarrollo sostenible en la gestión de los bosques, mostrando que la concepción que los entrevistados tienen acerca de la conservación y del desarrollo sostenible toma elementos de lo ya establecido en la norma e incorpora elementos adicionales más precisos, derivados de las intervenciones en el territorio (Tabla 2).

Tabla 2. Elementos que definen la conservación y el desarrollo sostenible, con base en la codificación y categorización de las respuestas de los entrevistados

\begin{tabular}{ll}
\hline Conservación & Desarrollo sostenible \\
\hline $\begin{array}{l}\text { Incluye diferentes estrategias de manejo, } \\
\text { como preservación estricta, restauración, } \\
\text { conocimiento y uso sostenible. }\end{array}$ & $\begin{array}{l}\text { Permite mantener los recursos naturales a través } \\
\text { del tiempo, para las futuras generaciones. }\end{array}$ \\
\hline $\begin{array}{l}\text { Debe buscar la convivencia del ser humano } \\
\text { con otras formas de vida y con el entorno. }\end{array}$ & Implica bienestar y prosperidad general. \\
& $\begin{array}{l}\text { Incluye la generación de crecimiento } \\
\text { económico. }\end{array}$ \\
$\begin{array}{l}\text { Busca mantener la estructura y el } \\
\text { funcionamiento de los ecosistemas y la } \\
\text { provisión de servicios ecosistémicos. }\end{array}$ & $\begin{array}{l}\text { Implica un equilibrio entre lo social, lo } \\
\text { ambiental y lo económico. }\end{array}$ \\
\hline
\end{tabular}

Se contempla, por ejemplo, el dinamismo de los ecosistemas y la necesidad de mantener su funcionamiento correcto para proveer bienes y servicios a las comunidades humanas; estas últimas, concebidas como parte integral de los ecosistemas. De manera similar, dentro del desarrollo sostenible se incorpora, además del crecimiento económico y de la conservación de los recursos para las generaciones futuras, la importancia del bienestar humano, como lo reflejan algunas de las respuestas de los entrevistados:

Es un concepto muy amplio que incluye elementos más integrales. Por ejemplo: la paz, el cambio climático, la conservación de la biodiversidad, el tema de género, la justicia. Hoy en día es más amplio que el concepto de Brundtland («Diego», 2018). 
Es lograr un bienestar humano. Podriamos ligarlo normativamente a un hecho que menciona la Constitución Nacional que es mantener la prosperidad general; y en esa prosperidad general, tal como lo reconoce la ley 165 del 94, reconocer que eso se le debe también a las otras especies ("Camilo», 2018).

Incluye no solo el crecimiento económico, sino el crecimiento social y la conservación del ambiente para las futuras generaciones. Es un desarrollo mucho más integrado, en el que va de la mano lo social, lo económico, lo cultural y lo ambiental (José», 2018).

Lo anterior pone en evidencia que, aunque no todos los entrevistados aportaron las mismas respuestas, el conjunto de elementos mencionados por ellos para definir desarrollo sostenible y conservación, incluye varios elementos de los que presentaron Berke y Conroy (2000), quienes definieron el desarrollo sostenible como «un proceso dinámico en el que las comunidades anticipan y adaptan las necesidades de las generaciones actuales y futuras, de modo que reproducen y equilibran los sistemas locales, sociales y económicos y vinculan las acciones locales a los temas mundiales» (p. 23); y quienes, además, plantearon principios para realizar el análisis del concepto en la planificación estatal de Estados Unidos, entre los que se incluyen: armonía con la naturaleza, economía local y equidad.

Por lo que respecta a la incorporación y puesta en marcha del concepto «desarrollo sostenible» desde la planificación, son diversos los elementos que los funcionarios y contratistas del MADS tienen en cuenta al momento de diseñar una propuesta:

- Se busca que las actividades a implementar en los proyectos no afecten el ecosistema.

- Se tiene presente las necesidades de las comunidades locales en términos de ingresos y seguridad alimentaria.

- Se fomenta la participación de la comunidad y se procura la incorporación de sus visiones e ideas.

- Se promueve la generación de conocimiento y el aprovechamiento de la biodiversidad del bosque sin talarlo, a partir de productos no maderables.

- Se incluye una sección de antecedentes y un marco normativo, lo cual visibiliza los compromisos internacionales que Colombia ha adquirido en términos de desarrollo sostenible.

La incorporación del marco normativo es fundamental para tener presente la existencia de los compromisos y metas a los que el MADS debe contribuir desde su quehacer y, así, direccionar adecuadamente las intervenciones. La verificación de los efectos probables de los proyectos sobre los ecosistemas es, igualmente, un componente crucial en el accionar del MADS, por cuanto una de sus funciones es generar directrices para garantizar la conservación de los ecosistemas. Por último, el componente de participación comunitaria es crucial para fortalecer la apropiación de los proyectos 
y la generación de acuerdos conducentes a la sostenibilidad de las intervenciones a través del tiempo.

De acuerdo con lo anterior, resulta conveniente retomar dos elementos ya mencionados: la definición de Brundtland sobre desarrollo sostenible — que establece el "qué» pero no el «cómo»—; y la visión institucional del MADS, que presenta los primeros esbozos del «cómo» cuando plantea temas como el ordenamiento integral del territorio, la educación ambiental y la articulación con el sector productivo. En este punto se hace visible que son los miembros de la organización — los funcionarios y contratistasquienes traducen el concepto para llevarlo de la teoría a la práctica. Así pues, cuando se plantea un proyecto, además de verificar que se cumpla con la normativa ambiental vigente, se contempla un trabajo articulado con las comunidades locales de modo tal que ellas contribuyan con la conservación de los ecosistemas (concibiendo la conservación como una diversidad de estrategias de manejo que incluyen la preservación y el uso) a la vez que mantienen sus prácticas culturales.

Sin embargo, aunque desde el MADS el concepto sea integral y se enfoque en la conservación de los bosques y sus servicios ecosistémicos a la vez que respeta los medios de vida de los habitantes del bosque, el modelo actual de desarrollo «implica la degradación continua y la conversión de bosques y otros hábitats naturales para la producción de cultivos agrícolas y actividades mineras» (MADS, 2015, p. 14). De hecho, las principales causas de deforestación en Colombia son la expansión de la frontera agropecuaria, la extracción ilícita de minerales, la expansión de la infraestructura, la extracción de madera y los incendios forestales (Cubillos, Chadid, Cubillos, Arias, Zúñiga, Joubert, Pérez y Berrío, 2017); los cuales son impulsados por diversos factores tales como el mercado, los incentivos estatales, las políticas sectoriales, el conflicto armado, las prácticas culturales y las características biofísicas del territorio, entre otros aspectos. Todo esto refleja la complejidad de las dinámicas territoriales y expone la dificultad para reducir la deforestación a cero dentro de los próximos dos años.

De acuerdo con lo anterior, la Estrategia Integral de Control a la Deforestación y Gestión de los Bosques «Bosques Territorios de Vida» (MADS, 2018a) plantea nueve principios orientadores de las actividades encaminadas a reducir la deforestación en el país, partiendo de la siguiente premisa:

una estrategia orientada a la reducción la deforestación y degradación de los bosques, enfrenta el desafío de construir una visión compartida a partir de las diferencias, que reconozca su significado y valor, es decir, una apropiación conjunta integrando las particularidades y reconociendo las diferencias (MADS, 2018a, p. 27).

De manera similar, aunque la Estrategia no presenta una definición específica de desarrollo sostenible, sí plantea que las actividades económicas que se lleven a cabo en busca del desarrollo del país deberán ser formuladas con base en la oferta natural 
y cultural del territorio, para generar bienes y servicios con valor ecosistémico y cultural. Lo anterior, teniendo en cuenta que los bosques requieren abordajes diferenciados según sus características ecosistémicas y su relación con las comunidades locales.

En este contexto, a través del programa Visión Amazonía, se pretende alcanzar en 2020 la meta de deforestación neta cero en la Amazonía colombiana; para lo cual trabajará de manera articulada con el Instituto SINCHI, Parques Nacionales Naturales, el IDEAM, las CAR, el MADR y las comunidades locales organizadas y no organizadas (étnicas y campesinas). Con este propósito, el Programa está estructurado en torno a cinco pilares: 1) el mejoramiento de la gobernanza forestal, 2) el desarrollo y planeación sostenible sectorial, 3) lo agroambiental, 4) la gobernanza ambiental con los pueblos indígenas y 5) las condiciones habilitantes. De esta manera, se busca contribuir con el fortalecimiento institucional para la gestión de los bosques; fortalecer espacios de diálogo con diversos actores vinculados a la cadena forestal; mejorar los instrumentos de ordenamiento ambiental en la región amazónica; promover sistemas productivos sostenibles que incluyan productos maderables y no maderables del bosque; mejorar las cadenas productivas y el acceso al mercado de varios productos; fortalecer la capacidad de los pueblos indígenas para la conservación de los bosques y recuperar sus tradiciones de producción sostenible; y consolidar un Sistema de Monitoreo de Bosques y Carbono y el Inventario Forestal Nacional (MADS, 2015).

De acuerdo con lo expuesto anteriormente, el Programa Visión Amazonía incorpora varios de los ODS; lo cual es coherente con las respuestas de los entrevistados a la pregunta sobre los ODS que hacen parte de la estrategia para el control de la deforestación en la Amazonía. Si bien la totalidad de los entrevistados reconoció que el ODS 15 «vida de los ecosistemas terrestres» es el que está directamente relacionado con las intervenciones para controlar la deforestación, otros (como acción por el clima, producción y consumo responsable, trabajo decente y crecimiento económico e igualdad de género) también pueden verse reflejados en las intervenciones.

Desafortunadamente, pese a la incorporación de diversas visiones en la formulación del programa y a la búsqueda de la implementación de acciones específicas para cada territorio, de manera articulada con la comunidad, hay diversos factores que dificultan el éxito de las intervenciones y que, por consiguiente, reducen las posibilidades de lograr la meta establecida para 2020. Al respecto, los entrevistados permitieron identificar que existen dificultades con diferente origen: unas se deben a debilidades en la estructura y operación propias del MADS y otras corresponden a factores externos.

Por ejemplo, la presencia del Estado en los territorios es insuficiente, lo cual dificulta ejercer labores de control, vigilancia y asistencia técnica; tal como ocurre en Caquetá "que tiene casi 9 millones de hectáreas, con una CAR que tiene 15 personas" ("Javier», 2018). Otro factor importante es la contradicción entre las políticas sectoriales o entre las visiones de desarrollo de los departamentos y la visión del MADS, 
tal como sucede en Guaviare, donde el discurso del gobernador actual refleja que su interés para fomentar el desarrollo del departamento es potenciar la ganadería y la producción de leche, lo cual contradice las intenciones de conservar los bosques y de detener la ampliación de la frontera agropecuaria. Sumado a lo anterior, existe una situación de orden público que restringe las intervenciones en algunos territorios, tal como sucedió en Guaviare cuando en el marco del pilar 1 de Visión Amazonía se intentaba desarrollar un proyecto de manejo forestal sostenible con las comunidades locales. De acuerdo con «Javier» (2018):

(...) una mezcla entre disidencia de la guerrilla y algunos actores interesados en deforestar (...) nos sacaron físicamente de un proyecto que estábamos desarrollando en el Guaviare. Imposible cómo ver unos pocos personajes (...), asustar a la gente y sacar un proceso que llevábamos meses construyendo con la comunidad; y en el tema de forestería comunitaria. Para mí eso fue demoledor.

\section{Discusión}

El concepto de desarrollo sostenible se encuentra explícito en los instrumentos de planificación del MADS y, a través de su operativización dentro de la entidad, ha sido alimentado con múltiples elementos que les han permitido a los funcionarios del MADS adaptarlo a las particularidades de cada territorio. Si bien el MADS maneja la definición establecida en el Artículo 3 de la Ley 99 de 1993, los instrumentos que ha generado reflejan que para la entidad el desarrollo sostenible abarca asuntos sociales, económicos, culturales y biofísicos que deben conducir, al mismo tiempo, a la conservación de los servicios ecosistémicos y al bienestar de las comunidades humanas. Esto resulta evidente en las respuestas de los entrevistados y en los documentos analizados, los cuales incluyen términos que van más allá de la conservación de la biodiversidad; incorporando, dentro de los elementos relacionados con el desarrollo sostenible, aspectos como la calidad de vida, la economía local, la gobernanza, la cultura y el conocimiento tradicional.

De acuerdo con lo anterior, podría decirse que para el MADS el desarrollo sostenible es aquel que permite mantener los recursos naturales a través del tiempo, por medio de acciones de preservación y restauración complementadas con la implementación de prácticas productivas diseñadas acorde con los intereses y necesidades de las comunidades, que respetan la resiliencia de los ecosistemas y el saber local; y que, al mismo tiempo, contribuyen con el bienestar y la prosperidad general. Por consiguiente, también es posible afirmar que la conceptualización de desarrollo sostenible de los profesionales del MADS se enmarca en el postdesarrollo, por cuanto visibiliza las formas de conocimiento producidas por quienes son los «objetos» del desarrollo para que puedan transformarse en sujetos y agentes (Escobar, 2005). En consecuencia, 
el diseño e implementación de modelos de uso sostenible de la biodiversidad y los servicios ecosistémicos con las comunidades locales constituye un mecanismo viable para controlar la deforestación en la Amazonía colombiana, siempre y cuando se controlen otros factores como la presencia de grupos armados al margen de la ley y el acaparamiento de tierras por actores foráneos.

Por ejemplo, la búsqueda de alternativas de uso del bosque en pie que incorporen el conocimiento local puede contribuir a la generación de productos con valor agregado conducentes al desarrollo endógeno y al bienestar de las comunidades (Vázquez Barquero, 2007), al mismo tiempo que se reducen las presiones sobre estos ecosistemas debido a la actividad agropecuaria. De acuerdo con el planteamiento de "Ana» (2018) «si la gente no cumple sus necesidades básicas va a tener que echar mano de lo que haya»; por lo cual, las propuestas de intervención en el territorio deben tener en cuenta las necesidades de las comunidades locales y prever mecanismos para que las satisfagan. Así mismo, se abre la posibilidad de vincular a un mayor número de miembros de la comunidad que no requieren habilidades específicas para el aprovechamiento de madera pero que posean otras habilidades, incluyendo a las mujeres, cuya participación actual en los procesos de gobernanza forestal es baja alrededor del mundo (Mai, Mwangi y Wan, 2011; Coleman y Mwangi, 2013). Tal como lo afirmó «Ana» (2008) «el bosque no se puede ver solo como bosque. Es receptor de vida, es regulador hídrico», lo cual pone de manifiesto que desde el MADS existe un reconocimiento del bosque como proveedor de multiplicidad de servicios ecosistémicos que van más allá de la producción de madera.

$\mathrm{Al}$ respecto, los programas como Visión Amazonía constituyen un mecanismo valioso en la senda hacia el desarrollo sostenible, por cuanto incorporan múltiples visiones y se construyen desde la base, incrementando las posibilidades de apropiación por parte de las comunidades y, por consiguiente, su pervivencia. La acción institucional del MADS, a través de este Programa, incide en el cambio de conducta de los habitantes locales que son usuarios del bosque; todo ello porque se aleja del paradigma de «no tocar» como única opción de conservación y permite la valoración de los bosques porque son fuente de numerosos beneficios, tanto cuando se aprovechan de manera directa como cuando se mantienen en pie. Esto resulta evidente en el pilar agroambiental del Programa, que impulsa la implementación de modelos agroambientales; en el pilar de gobernanza forestal, que propone esquemas de aprovechamiento forestal sostenible del bosque natural; y en el pilar indígena, que busca la incorporación de los saberes tradicionales en la conservación y gobernanza de los bosques. De manera similar, es evidente en propuestas como el desarrollo e implementación de un Programa de forestería comunitaria basado en la asociatividad y cadenas de valor de bienes y servicios del bosque, incluido en la EICD. 
Desafortunadamente, pese a ser un Programa con un planteamiento robusto, que ha presentado avances en la formulación de proyectos comunitarios con asociaciones indígenas; en la implementación de proyectos en sistemas productivos sostenibles con campesinos (MADS, s.f.); y en la conformación de dos redes de vigías rurales para la prevención y detección temprana de incendios forestales en los departamentos de Caquetá y Guaviare (MADS, 2018b); el incremento de la deforestación en la Amazonía genera incertidumbre acerca del logro de la meta de reducción de la deforestación y, por consiguiente, de la efectividad de la intervenciones.

Esta incertidumbre, además, se relaciona con las múltiples dificultades identificadas para gestionar el territorio amazónico, algunas de las cuales son ajenas a la gestión interna del MADS, como se presenta en la Tabla 3:

\section{Tabla 3. Dificultades para la reducción de la deforestación en la Amazonía y el logro del desarrollo sostenible}

Amenazas (factores externos)

Existe desarticulación y contradicción entre la visión de desarrollo sostenible del MADS y la visión de desarrollo de otros sectores, como es el caso de agricultura.

La situación de orden público impide la implementación de proyectos con las comunidades, pues grupos al margen de la ley restringen el ingreso de los equipos que pretenden realizar las acciones en los territorios o desaniman a las comunidades a participar.

El acaparamiento de tierras implica la praderización, promoviendo el crecimiento de la actividad ganadera y procesos de especulación en el mercado de tierras.

La falta de formalización de la propiedad dificulta al Estado realizar inversiones y también dificulta al campesino acceder a créditos.

La estructura jurídica y la fragmentación de competencias entre diversas entidades dificultan la puesta en marcha de proyectos integrales.

La presencia institucional en territorio es débil, lo cual es una consecuencia de la falta de recursos.
Más allá de los indicadores asociados a cambio de cobertura boscosa y captura de Carbono, los proyectos no incorporan indicadores de impacto (monitoreo) que permitan evaluar la eficiencia de las intervenciones en diferentes esferas: social, económica y ambiental.

La estrategia de planeación de las inversiones por vigencias dificulta garantizar los recursos y las acciones por períodos prolongados, imposibilitando la implementación de procesos continuos.

No se cuenta con un sistema de información documental que recoja los resultados de la totalidad de intervenciones realizadas.

No hay lineamientos sociales que orienten las intervenciones de los procesos que maneja la Dirección de Bosques del MADS.

La ausencia del Inventario Forestal Nacional (IFN) y de la valoración integral de los bosques dificulta tomar medidas de ordenamiento territorial por cuanto se desconoce la composición y estado real de los bosques. 
Además, como se observa en la columna de debilidades de la Tabla 3, el MADS requiere ser fortalecido como organización para contar con la capacidad técnica y financiera que le permita lograr mayor presencia y permanencia en el territorio y, de esa manera, acompańar los procesos comunitarios y ajustarlos a las realidades territoriales en tiempo real. Adicionalmente, se requiere un fortalecimiento en la generación de información útil y en su manejo, de modo tal que esta provea datos confiables y oportunos sobre las inversiones realizadas y sobre el impacto de los proyectos. Esta información debe convertirse en un soporte para la generación de nuevos instrumentos de planificación ambiental orientados al desarrollo sostenible.

Sumado a lo anterior, para maximizar el impacto del MADS en las acciones orientadas al control de la deforestación, hace falta mayor articulación entre dependencias tanto en la formulación de instrumentos como en la implementación de acciones. Por ejemplo, en la DBBSE los procesos participativos en los territorios son apoyados por los equipos técnicos de ingenieros forestales y biólogos sin un respaldo o lineamiento que desde la Subdirección de Educación y Participación (SEP) podría brindarse, particularmente en los procesos que implican trabajo con comunidades rurales. Por consiguiente, para garantizar la participación de las comunidades locales, resulta perentorio la generación de lineamientos sociales que optimicen los espacios de diálogo y concertación con los campesinos, los indígenas, los afros y demás actores presentes en los territorios con presencia de bosques. Estos espacios deben ser disenados y liderados por profesionales expertos en procesos de diagnóstico participativo, diseño de planeas de vida y, en general, de trabajo con comunidades organizadas y no organizadas. Lo anterior concuerda con lo que mencionó «Javier» (2018): «En la participación está el quid. Ahí está la salida, ahí está el camino».

Igualmente se requiere trabajo conjunto entre las áreas técnica, jurídica y presupuestal para que sea posible desarrollar procesos continuos con las comunidades. Al respecto, «Luisa» (2018) afirmó: «como ministerio somos muy fracturados: a nivel técnico soñamos y a nivel jurídico se aterriza qué se alcanza a hacer. Cosas que se planificaron en campo, luego no se pueden ejecutar por temas de jurídicos, de vigencias presupuestales...». Esto resultó evidente inicialmente en las respuestas de los entrevistados a la pregunta sobre la existencia de articulación entre las dependencias del MADS para formular los proyectos; a lo que la totalidad de las respuestas se redujo a «no» o "un poco». La falta de articulación puede influir de manera negativa en el éxito de los nuevos proyectos y normas porque las lecciones aprendidas de las implementaciones en territorio y de las propuestas generadas durante los proyectos quedan restringidas a una sola dirección o dependencia (e, incluso, a un solo grupo dentro de la dirección) $y$ pueden generar mensajes contradictorios entre instrumentos.

Por ejemplo, dentro del esquema de financiación de la EICD se incluyen los Pagos por Servicios Ambientales (PSA), los cuales corresponden a un «incentivo económico 
(...) que reconoce las acciones y las prácticas asociadas a la preservación y restauración de ecosistemas, que permiten minimizar conflictos en el uso del suelo y así favorecer el mantenimiento y la generación de servicios ambientales» (DNP, 2017). Sin embargo, mientras la EICD (que fue liderada por la Dirección de Bosques del MADS) enfatiza en la importancia de las prácticas culturales y del uso sostenible del bosque, planteando medidas como el desarrollo e implementación de un programa de forestería comunitaria, y de modelos agroambientales en las franjas de estabilización rural de la frontera agropecuaria; el Decreto 1007 de 2018 sobre PSA (que fue liderado por la Oficina de Negocios Verdes y Sostenibles del MADS) establece que las acciones a reconocer con los PSA son únicamente las de preservación y restauración de coberturas naturales. Además, direcciona la implementación de PSA hacia las áreas con coberturas naturales o potenciales para la restauración ecológica, toda vez que el valor del incentivo para los PSA hídricos y los PSA de reducción y captura de gases efecto invernadero se calcula a partir de la superficie de coberturas naturales. Esto excluye de manera drástica a aquellos propietarios cuyos predios cuentan con poca o ninguna cobertura natural y, por consiguiente, dificulta la implementación de muchas de las prácticas productivas sostenibles que impulsa la EICD y que se encuentran incluidas en el Plan Nacional de Negocios Verdes.

En lo relacionado con la articulación interinstitucional que actualmente realiza el MADS, se evidencia que está orientada a lograr incidencia en las políticas de Estado y en otros instrumentos de planificación que se generen. Por ejemplo, el relacionamiento con las entidades del gobierno nacional, que principalmente ocurre a través de espacios de diálogo denominados «mesas interinstitucionales», posibilita (aunque no garantiza) la articulación de proyectos y metas de desarrollo, así como la búsqueda de puntos de acuerdo para lograr el equilibrio entre las necesidades de un desarrollo económico y las prioridades de conservación de la biodiversidad a diferentes escalas. También permite identificar posibles sinergias para mejorar la efectividad de las intervenciones en territorio, de acuerdo con las particularidades de cada uno.

No obstante, se requiere que los espacios de articulación sean fortalecidos y operativizados para generar una estrategia de incidencia nacional que, articulada con otras entidades, sectores y fuentes de financiación, atenúe los numerosos factores externos a la gestión propia del MADS que están impidiendo el éxito de las intervenciones y que ponen en riesgo la meta planteada.

Si bien con la creación de la CICOD se generó una subdirección técnica de seguridad con participación de las fuerzas militares, es importante que las intervenciones sean oportunas y permanentes y que se enfoquen en los principales agentes de la deforestación. Al respecto, «Javier» (2018) afirmó que en el departamento del Guaviare las fuerzas militares realizan decomisos de madera que no son significativos en un entorno en donde claramente hay agentes deforestadores más poderosos que afectan grandes extensiones de tierra. Afirma el mismo entrevistado que con esos pequeños 
decomisos, las fuerzas militares «sacan pecho» pero en realidad no están atacando el problema de fondo.

De manera similar, resulta perentorio adelantar una estrategia de trabajo coordinada con otros sectores para que se apropien del tema ambiental como parte del desarrollo de su propio sector, comprendiendo que la responsabilidad de la conservación ambiental no es exclusiva del MADS. Tal como lo afirmó «Ana» (2018), refiriéndose a las metas del sector agricultura y desarrollo rural en comparación con las metas del sector ambiente, «lo que son metas para ellos, puede que para nosotros sean desastres». Esto es evidente cuando las metas de los gobiernos departamentales priorizan el desarrollo productivo sin contemplar lo ambiental y, por ejemplo, generan incentivos y subsidios para fomentar la producción de carne, leche o algún producto agrícola. Este tipo de políticas podrían incrementar las tasas de deforestación y se oponen a las propuestas enmarcadas en la agroecología y la explotación sostenible de recursos maderables y no maderables del bosque que lidera actualmente el MADS.

Por último, es importante tener en cuenta que el Programa Visión Amazonía constituye un referente para la formulación de estrategias de gestión participativa del territorio orientadas al control de la deforestación, que permitirá generar aprendizajes para fortalecer futuras propuestas. Desde luego, esto no significa que sea aplicable a todas las regiones; por lo que se requiere desarrollar el trabajo de diagnóstico y generación de propuestas con las comunidades de los nuevos territorios en donde se desee incorporar algún mecanismo de desarrollo sostenible y en donde se busque reducir la deforestación o la afectación a los ecosistemas. Al respecto, de acuerdo con Gertler (1997, citado por Rodríguez-Posé, 2013) las instituciones no solo moldean o direccionan, sino que también están modeladas por el entorno. Así, las instituciones se vuelven específicas para cada lugar, compartiendo características comunes a través de los territorios, pero también adoptando elementos particulares del propio ambiente institucional de cada territorio. Esto se articula con la propuesta de uno de los entrevistados («Luisa», 2018), quien sugirió que "para superar los cuellos de botella que dificultan la efectividad de las acciones, se requiere una formulación [de programas y proyectos] diferenciada por ecorregiones o sectores del país».

\section{Conclusiones}

Las evidencias presentadas a lo largo del documento permiten afirmar que el MADS está ejerciendo su rol como institución al avanzar significativamente en la formulación de lineamientos que conduzcan a la reducción de la deforestación en la Amazonía a través de la implementación de esquemas de uso sostenible de la biodiversidad, respetando los medios de vida de las comunidades que habitan los territorios boscosos e incorporando diversas visiones en la gestión del territorio. Adicionalmente, el relacionamiento con diferentes actores tanto del Estado como sociedad civil constituye un paso importante 
en la gestión orientada hacia el desarrollo sostenible; por cuanto la participación, la concertación, las alianzas y la generación de espacios inclusivos y orientados hacia el bienestar de las comunidades locales, incorporan elementos de varios ODS.

Sin embargo, aún se requiere trabajar en el fortalecimiento de la operatividad del MADS como organización. Dado que, pese a contar con una estructura de operación por procesos que orienta las actividades, hace falta una ruta que incluya espacios de consulta y articulación entre dependencias; de modo tal que la formulación e implementación de políticas, planes y proyectos recoja los aprendizajes de las experiencias previas implementadas por las diversas dependencias de la entidad.

No obstante, más allá del fortalecimiento del MADS, se requieren cambios estructurales en las políticas de desarrollo colombianas, que conduzcan al posicionamiento del sector ambiental como elemento constitutivo de los procesos de toma de decisiones. Además, es necesario que los factores externos que afectan la gestión institucional propia del MADS, como es el caso de los problemas de orden público y de tenencia de la tierra, sean controlados de manera efectiva por la fuerza pública.

Por último, debido al dinamismo en la generación de instrumentos normativos orientados al control de la deforestación en Colombia, es recomendable analizar en futuros estudios la efectividad de cada instrumento en términos de pertinencia, facilidad de implementación e impacto sobre el territorio y sobre las comunidades rurales que hoy habitan las áreas con las mayores tasas de deforestación en Colombia.

\section{REFERENCIAS}

Alpuche de la Cruz, E. y Bernal, J. (2015). La institución y la organización: un análisis centrado en el actor. Intersticios Sociales, (10), 1-29.

Avendaño, C., Fonseca, M. y Marín, D. (2011). Estructura organizacional y su relación con la efectividad de los servicios universitarios de atención psicológica. Psychol. Av. Discip. 5(2), 33-44.

Barrera, L., Bedoya, J. y Rodríguez, J. (2007). Creando las bases conceptuales para un análisis institucional de los ministerios en Colombia. Bogotá: ESAP.

Berke, P. y Conroy, M. (2000). Are We Planning for Sustainable Development? An Evaluation of 30 Comprehensive Plans. Journal of the American Planning Association 66(1): 21-33. https://doi.org/10.1080/01944360008976081

Chevallier, J. (1996). Institutions politiques. Droit et Societé, 33: 485-502

Coleman, E. y Mwangi, E. (2013). Women's participation in forest management: A cross-country analysis. Global Environmental Change, 23(1), 193-205. https://doi. org/10.1016/j.gloenvcha.2012.10.005

Constitución Política de Colombia (1991). Asamblea Nacional Constituyente. Colombia. Corte Suprema de Justicia (2018). Sentencia STC4360-2018. Colombia. 
Creswell, J. W. (2013). Qualitative inquiry and research design: Choosing among five approaches. Thousand Oaks, CA: Sage.

Creswell, J. W. (2014). Research design: Qualitative, quantitative, and mixed methods approaches. Thousand Oaks, CA: Sage.

Dayer, A., Lutter, S., Sesser, K., Hickey, C. y Gardali, T. (2017). Private Landowner Conservation Behavior Following Participation in Voluntary Incentive Programs: Recommendations to Facilitate Behavioral Persistence. Conservation Letters, 12(2), 1-11. https://doi.org/10.1111/conl.12394

Departamento Administrativo de la Función Pública (23 de septiembre de 2011). Decreto 3570 de 2011. Bogotá.

Departamento Nacional de Planeación - DNP (2014). Bases del Plan Nacional de Desarrollo 2014-2018. Versión para el Congreso.

Departamento Nacional de Planeación - DNP. (2017). CONPES 3886. Lineamientos de política y programa nacional de pago por servicios ambientales para la construcción de paz. Recuperado de: https://bit.ly/2uGGOwN

Departamento Nacional de Planeación - DNP. (2018a). Documento CONPES 3918. Estrategia para la implementación de los Objetivos de Desarrollo Sostenible (ODS) en Colombia. Recuperado de https://bit.ly/2tT4nFf

Departamento Nacional de Planeación - DNP. (2018b). Documento CONPES 3934. Política de Crecimiento Verde. Recuperado de https://bit.ly/2mdcc1L

Ehrlich, P.R. (1989). The limits to substitution: Meta resource depletion and new economicecological paradigm. Ecological economics, 1(1), 1-10. https://doi.org/10.1016/09218009(89)90021-9

Escobar, A. (2005). El «postdesarrollo» como concepto y práctica social. En Daniel Mato (coord.), Políticas de economía, ambiente y sociedad en tiempos de globalización (pp. 17-31). Caracas: Facultad de Ciencias Económicas y Sociales, Universidad Central de Venezuela.

Escobar, A. (2011). Sustainability: Design for the pluriverse. Development, 54(2), 137-140. https://doi.org/10.1057/dev.2011.28

Escobar, A. (2015). Decrecimiento, post-desarrollo y transiciones: una conversación preliminar. Interdisciplina, 3(7), 217-244. https://doi.org/10.22201/ ceiich.24485705e.2015.7.52392

González, J., Cubillos, A., Chadid, M., Cubillos, A., Arias, M., Zúńiga, E., Joubert, F. Pérez, I. y Berrío, V. (2017). Caracterización de las principales causas y agentes de deforestación a nivel nacional periodo 2005-2015. Bogotá: Instituto de Hidrología, Meteorología y Estudios Ambientales - IDEAM. Ministerio de Ambiente y Desarrollo Sostenible. Programa ONU-REDD Colombia.

Gulick, L. (1993). Notas sobre la teoría de la organización. En Teoría de la organización (vol. 1, pp. 113-127). Madrid: Ministerio para las Administraciones Públicas de Madrid. 
Instituto de Hidrología, Meteorología y Estudios Ambientales - IDEAM (2017). Resultados monitoreo de la deforestación 2017. Recuperado de https://bit.ly/2Q8MHfm

Instituto de Hidrología, Meteorología y Estudios Ambientales - IDEAM (2018). Décimo tercer boletin de alertas tempranas de deforestación (AT-D) cuarto trimestre 2017. Recuperado de https://bit.ly/2KX517W

Instituto de Hidrología, Meteorología y Estudios Ambientales - IDEAM (2018). IDEAM presentó los datos actualizados del monitoreo a la deforestación en 2017. Recuperado de: https://bit.ly/2QmYdUx

Instituto Amazónico de Investigaciones Científicas Sinchi (2016). Orientaciones para reducción de la deforestación y degradación de los bosques. Recuperado de https:// bit.ly/2wCzHJU

Ley 99 de 1993. Congreso de Colombia (22 de diciembre de 1993)

Ley 1776 de 2016. Congreso de Colombia (29 de enero de 2016)

Mai, Y., Mwangi, E. y Wan, M. (2011). Gender analysis in forestry research: looking back and thinking ahead. International Forestry Review, 13(2), 245-258 https://doi. org/10.1505/146554811797406589

Martín-Crespo, M. y Salamanca, A. (2007). El muestreo en la investigación cualitativa. Nure Investigación, 27, marzo-abril 07.

Ministerio de Agricultura y Desarrollo Rural - MADR (2018). Resolución 261 de 2018. Por medio de la cual se define la frontera agrícola nacional y se adopta la metodología para la identificación general (21 de junio de 2018).

Ministerio de Ambiente y Desarrollo Sostenible - MADS (2012). Política Nacional para la Gestión Integral de la Biodiversidad y sus Servicios Ecosistémicos - PNGIBSE. Bogotá.

Ministerio de Ambiente y Desarrollo Sostenible - MADS y Programa de las Naciones Unidas para el Desarrollo - PNUD (2014). Quinto Informe Nacional de Biodiversidad de Colombia ante el Convenio de Diversidad Biológica. Bogotá, D.C., Colombia.

Ministerio de Ambiente y Desarrollo Sostenible - MADS (2015). Ambiente y Desarrollo Sostenible. Recuperado de http://bit.ly/2HV8aa2

Ministerio de Ambiente y Desarrollo Sostenible - MADS (2016). Visión Amazonía. Presentación. Recuperado de https://goo.gl/pDX3dr

Ministerio de Ambiente y Desarrollo Sostenible - MADS (28 de febrero de 2017). Resolución 0470 de 2017.

Ministerio de Ambiente y Desarrollo Sostenible - MADS (25 de julio de 2017). Decreto 1257 de 2017. Bogotá.

Ministerio de Ambiente y Desarrollo Sostenible - MADS (10 de octubre de 2017). Decreto 1655 de 2017. Bogotá.

Ministerio de Ambiente y Desarrollo Sostenible - MADS (14 de junio de 2018). Decreto 1007 de 2018. Bogotá. 
Ministerio de Ambiente y Desarrollo Sostenible - MADS (2018a). Bosques Territorios de Vida. Estrategia Integral de Control a la Deforestación y Gestión de los Bosques. Bogotá.

Ministerio de Ambiente y Desarrollo Sostenible - MADS (2018b). Informe de gestión 2010-2018. Dirección de Bosques, Biodiversidad y Servicios Ecosistémicos. Bogotá.

Ministerio de Ambiente y Desarrollo Sostenible - MADS (s.f.). Avances Estrategia Integral de Control a la Deforestación y Gestión de los Bosques. Bosques territorios de vida. Recuperado de: https://bit.ly/2qKcBLm

Naciones Unidas (1973). Informe de la Conferencia de las Naciones Unidas sobre el Medio Humano. Estocolmo, 5 a 16 de junio de 1972. Recuperado de: https://bit.ly/2egVgqI

Naredo, J. (1987). La economía en evolución: historia y perspectivas de las categorías básicas del pensamiento económico. Ministerio de Economía y Hacienda, Secretaría de Estado de Comercio. España.

North, D. (1991). Institutions. The Journal of Economic Perspectives, 5(1), 97-112. https:// doi.org/10.1257/jep.5.1.97

Ostrom, E. (1990). Governing the commons: The Evolution of Institutions for Collective Action. Cambridge University Press. https://doi.org/10.1017/CBO9780511807763

Parada, L. y Sánchez, L. (2014). Desarrollo Sostenible en Colombia: una utopía, una necesidad del presente y un alivio para el futuro. Contexto, 181-189.

Rodríguez-Posé, A. (2013). Do institutions matter for regional development? Regional Studies, 47(7), 1034-1047 https://doi.org/10.1080/00343404.2012.748978

Sistema de Información Ambiental de Colombia - SIAC (2016). Cambio en la superficie cubierta por bosque natural 2016. Recuperado de https://bit.ly/2wDbQK0

Tropical Forest Alliance 2020 (2017). Alianza público privada cero deforestación TFA 2020 Colombia. Recuperado de https://bit.ly/2rKlB4o

Unesco (2015a). La Unesco y los Objetivos de Desarrollo Sostenible. Recuperado de https:// es.unesco.org/sdgs

Unesco (2015b). Desarrollo sostenible. Recuperado de https://goo.gl/Cv4UDu

United Nations (1987). Report of the World Commission on Environment and Development "Our Common Future». United Nations General Assembly Forty-second session.

United Nations (2015a). FAQs Sustainable Development Summit. Recuperado de https:// bit.ly/2Id3oqi

United Nations (2015b). The New York Declaration on Forests. Climate Summit 2014. Recuperado de https://bit.ly/2z6lq6S

Vázquez Barquero, A. (2007). Desarrollo endógeno. Teorías y políticas de desarrollo territorial. Investigaciones Regionales, 11, 183-210.

World Wildlife Fund (2015). Proyecto «Identificación de los motores, agentes y causas subyacentes de la deforestación en el departamento del Putumayo: Valle del Sibundoy, municipios de Villagarzón y Puerto Leguízamo». Convenio TW07 SINCHI-WWF. 